\title{
REDUCED 6-keto PGF1a AND PLASMA RENIN ACTIVITY IN PRE-ECLAMPTIC SUBJECTS
}

\author{
SHARMA R.C. ${ }^{1}$ AND SHARMA R.K. ${ }^{2}$ \\ 1Department of Physiology, Faculty of Medicine, School of Biomedical Sciences, AIMST University, Malaysia. \\ 2Department of Obstetrics and Gynecology, Padmashree Dr. D. Y. Patil Medical College, Mauritius. \\ *Corresponding Author: Email- drsharmax@gmail.com
}

Received: November 13, 2011; Accepted: April 09, 2012

\begin{abstract}
Prostacyclin, a potent vasodilator having anti-platelet aggregating properties, is known to play a role in regulating vascular tone and vasoconstrictor response to Angiotensin. In the present study plasma level of 6-keto prostaglandin $F$ as indicator of Prostacyclin activity (PGI2) and Angiotensin I as an indicator of plasma Renin Activity (PRA) in normal pregnant women and pre-eclamptic women were measured using radioimmunoassay. Prostacyclin production was significantly decreased in pre eclampsia compared with normal pregnant women. The results were analyze to establish co-relation with severity of disease and observed a strong negative correlation ( $r=-56)$ between PGI2 \& severity of disease, suggesting involvement of this Vasoactive prostaglandin in causation and the associated hypertension of this disorder. Key words- Preeclampsia, Plasma 6-keto Pgf1a Acivity, Plasma Renin Activity.
\end{abstract}

Citation: Sharma R.C. and Sharma R.K. (2012) Reduced 6-keto PGF1a \& Plasma Renin Activity in Pre-eclamptic Subjects. International Journal of Molecular Biology, ISSN: 0976-0482 \& E-ISSN: 0976-0490, Volume 3, Issue 1, 2012, pp.-40-42.

Copyright: Copyright@2012 Sharma R.C. and Sharma R.K.. This is an open-access article distributed under the terms of the Creative Commons Attribution License, which permits unrestricted use, distribution, and reproduction in any medium, provided the original author and source are credited.

\section{Introduction}

The role of prostaglandin in heamodynamic changes in fetal and maternal circulation has been reported by Terrango (1). Toxemia is chacterised by increased vasoconstriction frequently associated with increased platelet aggregation and reduced Uteroplacental blood flow. Because prostacyclin is a potent vasodilator and having anti-platelet aggregating properties, it can be modulated by local prostaglandin (2). In pregnancy-induced hypertension, prostacyclin production reported to be reduced or unchanged but its production has not been critically examined. Since preeclampsia is characterized by increased sensitivity to pressor effect of Angiotensin II, It is proposed to study the plasma level of 6-keto prostaglandin F as indicator of Prostacyclin activity (PGI2) and plasma Renin Activity (PRA) in normal pregnant women and pre-eclamptic women, with a aim to find out co-relation if any between the PGI2 \& pre-eclampsia.

\section{Material and Methods}

The material for the present study was drawn from the patient attending anti-natal clinic and those who were admitted in wards of Obstetric \& Gynecology of Teaching General Hospital. The subjects for the present study comprises of Normal non-pregnant women $(n=35)$, Normal Pregnant women with gestational age of 32 wk. or above $(n=35)$, and patients with pre-eclampsia $(n=35)$. The subjects for the present study were selected after thorough clinical examination e.g. P/A for gestational age, edema, and blood pressure. The subjects having personal/family history of hypertension, Diabetes, any medication were excluded from the study. The grading of the severity of the pre eclampsia was as per the classification described by Massani et al (3). Plasma Samples were taken from the ante-cubital vein after recording the blood pressure of the patient in supine position in ice freezed tube as per the method described in the protocol of 6-keto PGF and PRA estimation. The plasma was separated by centrifugation and stored in Deep freeze until assayed. The PGI2 (6-keto PGF1a) activity was estimated by measuring its stable metabolite $c$ in plasma using RPA 515 RIA kit supplied by Amersham International Plc. UK. The PR activity by generating Angiotensin I under controlled condition. The generated Angiotensin I is then quantitated by RIA using kit developed by BARC.

\section{Observations}

Plasma level of the 6-keto PGF1a and PRA were measured in a total of 105 cases and mean 6-keto PGF1a and PRA in normal 
non pregnant, normal pregnant and in cases of preeclampsia. It is observed that PGI2 activity (mean 6-keto PGF1a level) as well as Plasma renin Activity (PRA) in cases of pre-eclampsia were found to be significantly lower than that of pregnant women $(P<$. 001) and non pregnant group $(P<001)$. The Pregnant women were associated with higher level of PGI2 (6-keto PGF1a) and PRA as compared to non-pregnant and found to be statistically significant. Prostacyclin level (6-keto PGF1a) and PRA level in pre eclampsia were analyzed with severity of pre eclampsia to study the relationship between activity of PGI2 level \& PRA, it is observed that there is gradual decrease in PGI2 activity with severity of the diseases suggesting a inverse relation between PGI2 and severity. A similar relationship was observed between PRA and severity of the disease.

\section{Discussion}

Prostacyclin is a prostanoid synthesized from the endoperoxide in the fetal and maternal musculature. Unlike other prostaglandin, $P G I 2$ is not inactivated during its passage through pulmonary circulation, hence it is known to exert it effects on local as well on systemic vasculature. It has been reported that prostacyclin has a strong vasodilator effect $(4,5)$, partially mediated by attenuation of vasoconstriction caused by Angiotensin II $(6,7)$. Increase in urinary excretion of 6-keto PGF1a in normal pregnant women as compared to non-pregnant women has been reported by Goodman (8). Garber et al have observed high level of 6-keto PGF1a in uterine vein in dog. Increased production of prostacyclin in placenta (9), pregnant uterus (10) in human subject as reported, suggests that increased synthesis of prostacyclin in normal pregnancy is mainly from feto-placental vasculature. It is reported that PGE a potent vasodilator is found to increase during pregnancy as compared to normal pregnant subject. However its contribution in causing heamodynamic changes in normal pregnancy is less significant because of its rapid inactivation in pulmonary bed. Gant et al performed sequential determinations of pressor response to infusion of Angiotensin II in pregnant women and observed that women with normal pregnancy demonstrated decrease pressor effect of Angiotensin II therefore the blood pressure does not rise in pregnancy inspite of increased plasma renin activity (11). Patrono et al has demonstrated that PGI2 is synthesized in renal cortex and has direct stimulatory effect on the renin release mechanism (12). This has attributed to close proximity of JG apparatus vascular endothelium as vascular endothelium microsome produces large quantities of prostacyclin when incubated with endoperoxide (13).

In the present study we observed two fold increase in 6-keto PGF1a in normal pregnancy as compared to normal non-pregnant controls. These findings are in agreement with the reports of other workers $(8,14,15)$. In normal pregnancy, renin release is under the control of several factors such as renal perfusion, sodium intake, volume expansion etc. Prostaglandin synthesis is also known to a stimulus for renin release. Weber has demonstrated that in vivo incubation of arachidonic acid endoperoxide or prostacyclin with renal cortical slices of rabbit caused released of renin (16). Vascular sensitivity to Angiotensin II has been reported to be another determinant of renin secretion. Brunner has proposed that changes of intracellular sodium and calcium concentration in the vessel wall or synthesis of Angiotensin II antagonists such as prostacyclin in the blood vessel may be a factor to control vascular sensitivity and receptor affinity (17). In the present study, PRA was estimated by measuring Angiotensin I generated per ml per hour, which reflects the plasma renin activity and its substrate concentration. Elevation of PRA found in our study is in consistent with other reports $(18,19,20)$.

The observation of present study as regards to 6-keto PGF1a level in pre eclampsia are comparable with observations as reported by others (21-23) with slight difference in the level. This difference in the mean 6-keto PGF1a may be due to various factors such as technique of sampling of blood, extraction of PGF from plasma sample, racial difference and selection criteria of the subjects. However all the reports have observed reduced 6-keto PGF1a level in pre eclampsia ranges from $40.2 \%$ to $62.7 \%$. Our study has observed $60 \%$ decrease in 6-keto PGF1a level in pre eclampsia as compared to normal pregnant women. Reduced 6keto PGF1a level in pre eclampsia reported by others as well as in present study suggests that reduced production of prostacyclin has been attributed partly to limited availability of enzyme responsible for conversion of PGH2 in to PGI2. It has been shown that $\mathrm{PGH} 2$ conversion in to PGI2 require prostacyclin synthetase (24). Reduced oxygen availability to placental tissue resulting from uteroplacental ischaemia could also be linked in mechanism of defective prostacyclin production (15). Reduced arachidonic acid in fetal circulation may account for decreased production of prostacyclin in foeto-maternal unit. Reduced PRA in pre eclampsia indicates suppression of renin release mechanism in the kidney, which has been attributed to, decreased prostacyclin production or sodium \& water retention, a feature of pre eclampsia may suppress the release of renin from kidney and cause fall in PRA. Decreased concentration of renin substrate could also be another factor for suppression of PRA in preeclampsia (25). It was reported that retention of sodium and water in pre eclampsia is presumably due to decrease glomerular filtration of sodium which in turn is due to decreased in plasma sodium and reduced glomerular filtration rate seen in preeclampsia (24).

Very few attempts have been made to establish relationship with severity of the disease. In present study we observed a negative correlation between 6keto PGF1 and severity of the diastolic blood pressure $(r=-0.61)$ as well with systolic blood pressure $(r=-0.56)$, which suggest that fall in 6-keto PGF1a increases responsiveness of vasculature to the circulatory vasoactive hormones. The present study shows a strong correlation between PGI2 and PRA suggests that increase in circulation level of prostacyclin as measured by 6-keto PGF1a could explain not only generalized vasodilatation but also an increase in PRA. Low prostacyclin production on the other hand could explain the generalized vasoconstriction and depressed plasma renin activity in pre eclampsia.

Reduced prostacyclin level, as an indicator of prostacyclin production during pre eclampsia observed in the present study appears to play an important role in pathogenesis of pre eclampsia, though several factors have been reported to participate in the prostacyclin synthesis. It may however be speculated that an imbalance and interface with normal enzymatic activities during PGI2 synthesis may reduce its synthesis. Reduced prostacyclin in uterine vasculature may cause a reduction in uteroplacental blood flow. Lack of physiological changes in placenta and spiral artery lesion may also be induced by decreased production which In turn account for 
uteroplacental ischaemia. Reduced 6-keto PGF1a level associated with suppression of PRA in pre eclampsia support the evidence that renal prostacyclin synthesis pay a role in the renin secretion. Increased sensitivity of blood vessels to vasoconstrictor substances such as Angiotensin-II as compared to normal pregnancy is a characteristic feature of pre eclampsia, which is augmented when treated with prostacyclin inhibitors such as indomethacin and aspirin (25). Thus in pre eclampsia, a decrease in PGI2 synthesis in blood might increase the vascular smooth muscle responsiveness to Angiotensin-II, thereby making absolute level of Angiotensin-II less important than the sensitivity of vessel to Angiotensin-II and may cause a generalized vasoconstriction which is responsible for hypertension and generalized endothelial damage leading to proteinuria.

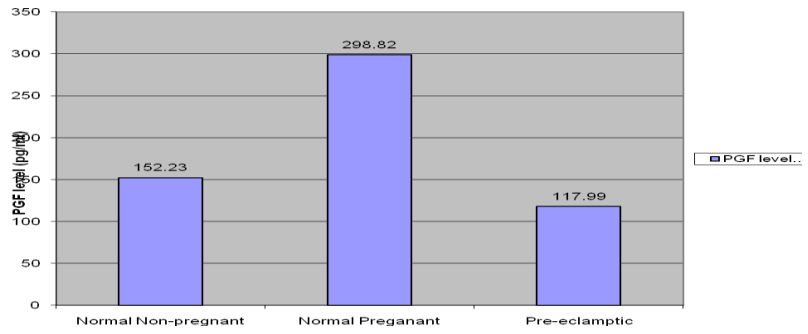

Chart I- PGF1a level in preeclampsia as compared to normal pregnant women and non-pregnant subject

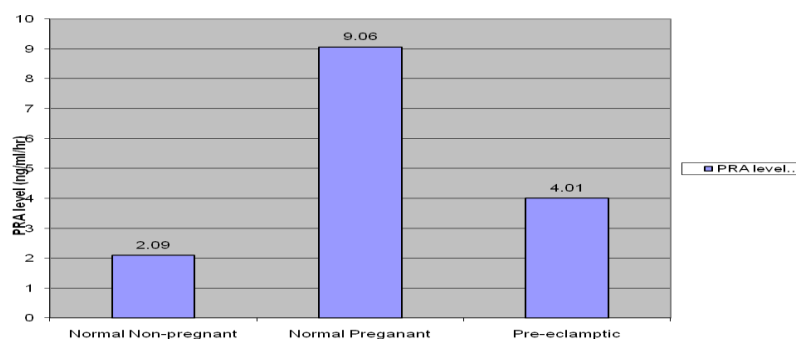

Chart II- PRA level in preeclampsia as compared to normal pregnant women and non-pregnant subject

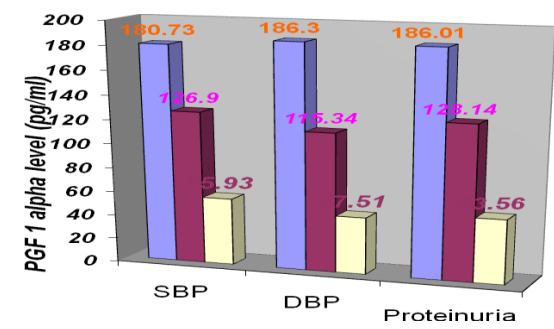

口 Mild

Chart III- Relationship between PGF1a level and severity of preeclampsia

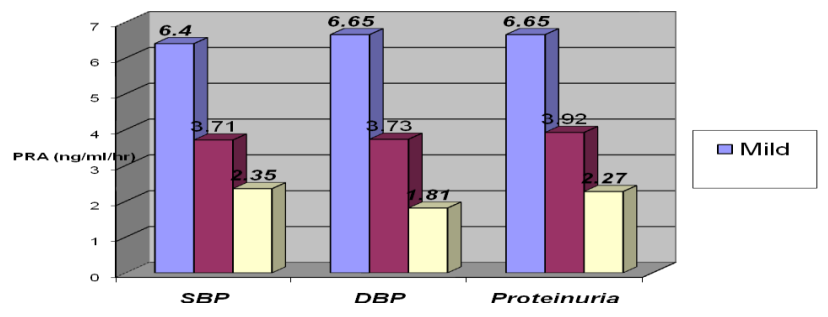

Chart IV- Relationship between PRA Level and severity of preeclampsia

\section{Conclusion}

We therefore conclude that deficient prostacyclin in pre eclampsia may play an important role in pathogenesis of pre eclampsia and provide a patho-physiologic explanation for the clinical features of the disease. However further studies to identify the genetic defect in vascular endothelium has been suggested.

\section{References}

[1] Terrango N.A., McGiff J.C., Murray S., Terrango A. (1978) Prostaglandin, 16, 847.

[2] Pomerantz K., Sintetose A. and Rawell P. (1987) Prostaglandin, 15, 1035.

[3] Needleman P. and Kaley G. (1997) New Eng. J. Med., 298, 1122.

[4] Massani K.M., Parikh M.N. (1996) Toxaemias of pregnancy in "textbook of obstetrics, 231.

[5] Armstrong J.M., Moncada S., Vane J.R. (1978) Br. J. Pharma., 65, 125.

[6] Weeks I.R., Compton L.D. (1979) Prostaglandin, 17, 501.

[7] Tada N., Miyazaki M. (1979) Am. J. Phy., 240(H), 249.

[8] Goodman R.P., Killam A.R., Brash A.R. (1982) Am. J. Obste. Gynec, 142, 817.

[9] Myatt L. and Elder M.G. (1977) Nature L, 159, 159.

[10]Omni C., Folco G.C., Fano M. and Berti F. (1979) Prostaglandin, 17, 113.

[11]Gant N.F., Worley R.J. (1998) Hypertension in pregnancy concept and management, 37, 198.

[12]Patrono C., Pugliese F., Ciabattoni G. (1982) J. Clin. Invest., 69,231

[13]Gryglewiski J.R., Zmuda A. (1978) Atherosclerosis, 31, 385.

[14]Lewis P.J., Boylan P., Downing I. (1980) Br. Med. J., 280, 1581.

[15]Walsh W.S. (1995) Am. J. Obste. Gynec., 152, 335.

[16]Larson C., Waber P., Anggard E. (1984) Eur. J. Pharma., 28, 391.

[17]Brown J.J., Davies D.L., Doak P.B. (1983) Lancet, 2, 900.

[18]Pederson E.B., Rasmussen A.B. (1982) Acta Endo, 101, 273.

[19]Weinberger M.H., Kranner N.J. and Peterson L.P. (1976) Hypertension in pregnancy, 271.

[20]Dieckmann W.J., Michel H.L. (1976) Am. J. Obste. Gynec., 33 (131), 1937.

[21]Walsh W.S. (1985) Am. J. Obste. Gynec., 152, 335.

[22]Downing I., Shepherd G.L., Lewis P.J. (1980) Lancet, 2, 1374.

[23]Weber P.C., Larson E., Anggard E., Hamburg M. (1976) Cir. Res., 39, 868.

[24]Gorden R.D., Persons S., Symonch E.M. (1996) Lancet, 1, 347.

[25]Leindheimer M.D., Kartz A.I. (1977) Kidney function and diseases in pregnancy Eds. Lea and Febiger Philadelphia, 82.

[26]Sheppard B.L. and Bonner J. (1976) J. Obste. Gynec. Br. Comm. 81, 497.

[27]Shebuski R.J., Aiken J.W. (1980) J. Cardio. Phar., 2, 667. 